\title{
COMPOSTO ORGÂNICO E INOCULAÇÃO MICORRÍZICA NA PRODUÇÃO DE MUDAS DE PINHÃO MANSO ${ }^{1}$
}

\author{
Jolimar Antonio Schiavo ${ }^{2}$, Cristiane Andreia da Silva ${ }^{2}$, Jean Sérgio Rosset ${ }^{2}$, \\ Mateus Luiz Secretti ${ }^{2}$, Ricelly Aline Camargo de Sousa ${ }^{2}$, Nanci Cappi ${ }^{2}$
}

\begin{abstract}
ORGANIC COMPOST AND MYCORRHIZAL

INOCULATION ON PHYSIC NUT SEEDLINGS

Physic nut species has shown great potential for biofuel production. This study aimed to evaluate the effect of inoculation with the arbuscular mycorrhizal fungus Glomus clarum and the application of organic compost on the growth and phosphorus (P) and nitrogen $(\mathrm{N})$ absorption, in physic nut seedlings. A randomized experimental blocks design, in a $5 \times 2$ factorial scheme, was used, with the evaluation of organic compost levels $\left(0 \mathrm{tha}^{-1}, 15 \mathrm{tha}^{-1}, 30 \mathrm{tha}^{-1}, 60 \mathrm{tha}^{-1}\right.$, and $\left.120 \mathrm{tha}^{-1}\right)$ and presence or absence of Glomus clarum, with four replications. Physic nut seedlings inoculated with Glomus clarum presented, at levels of $15 \mathrm{t} \mathrm{ha}^{-1}$ of organic compost, the lowest height (20\%), 120 days after sowing. However, the basal diameter increased, at levels of 30 t ha $^{-1}(21 \%)$ and 120 tha $^{-1}(33 \%)$ of organic compost, 60 days after sowing. Only in the treatment with Glomus clarum, physic nut seedlings showed increasing linear regression adjustment in the dry matter of stem $\left(\mathrm{R}^{2}=0.99\right)$, aerial part $\left(\mathrm{R}^{2}=0.98\right)$, roots $\left(R^{2}=0.95\right)$, and whole plant $\left(R^{2}=0.97\right)$, depending on the organic compost levels. Physic nut seedlings inoculated with Glomus clarum showed increases in P content of the aerial part of $93 \%, 37 \%, 57 \%$, and $51 \%$, for levels of $15 \mathrm{t} \mathrm{ha}^{-1}, 30 \mathrm{t} \mathrm{ha}^{-1}$, $60 \mathrm{t} \mathrm{ha}^{-1}$, and $120 \mathrm{t} \mathrm{ha}^{-1}$ of organic compost, respectively. For the $\mathrm{N}$ content, such increases were $50 \%$ and $45 \%$, at levels of $0 \mathrm{t} \mathrm{ha}^{-1}$ and $60 \mathrm{t} \mathrm{ha}^{-1}$ of organic compost, respectively.
\end{abstract}

KEY-WORDS: Jatropha curcas L.; biofuel; Glomus clarum; seedlings quality.

\section{INTRODUÇÃO}

Óleos vegetais provenientes de diversas oleaginosas têm sido testados com sucesso na produção de biodiesel. A espécie pinhão manso (Jatropha curcas $\mathrm{L}$.) tem sido considerada promissora para este fim, devido ao elevado teor de óleo, o qual varia de $25 \%$ a $40 \%$, superior ao encontrado na maioria das

\section{RESUMO}

A espécie pinhão manso têm se mostrado bastante promissora para a produção de biodiesel. O presente trabalho objetivou avaliar o efeito da inoculação do fungo micorrízico arbuscular Glomus clarum e da aplicação de composto orgânico sobre o crescimento e absorção de fósforo (P) e nitrogênio (N), em mudas de pinhão manso. Utilizou-se o delineamento experimental em blocos ao acaso, em esquema fatorial $5 \times 2$, sendo avaliados os fatores doses de composto orgânico ( $0 \mathrm{t} \mathrm{ha}^{-1}$, $15 \mathrm{t} \mathrm{ha}^{-1}, 30 \mathrm{t} \mathrm{ha}^{-1}, 60 \mathrm{t} \mathrm{ha}^{-1}$ e $120 \mathrm{tha}^{-1}$ ) e presença ou ausência do inoculante Glomus clarum, com quatro repetições. As mudas de pinhão manso inoculadas com Glomus clarum apresentaram, na dose de 15 tha $^{-1}$ de composto orgânico, menor altura (20\%), aos 120 dias após a semeadura. No entanto, o diâmetro, à altura do colo, foi maior, nas doses de $30 \mathrm{t} \mathrm{ha}^{-1}(21 \%)$ e $120 \mathrm{t} \mathrm{ha}^{-1}$ (33\%) de composto orgânico, aos 60 dias após a semeadura. Apenas no tratamento com Glomus Clarum, mudas de pinhão manso apresentaram ajuste de regressão linear crescente, na matéria seca de caule $\left(R^{2}=0,99\right)$, parte aérea $\left(R^{2}=0,98\right)$, raízes $\left(R^{2}=0,95\right)$ e planta inteira $\left(\mathrm{R}^{2}=0,97\right)$, em função das doses de composto orgânico. Mudas de pinhão manso inoculadas com Glomus clarum apresentaram incrementos nos teores de $\mathrm{P}$ da parte aérea de $93 \%, 37 \%, 57 \%$ e $51 \%$, para as doses de $15 \mathrm{t} \mathrm{ha}^{-1}, 30 \mathrm{t} \mathrm{ha}^{-1}$, 60 t ha $^{-1}$ e 120 t ha $^{-1}$ de composto orgânico, respectivamente. Para os teores de N, tal incremento foi de $50 \%$ e $45 \%$, nas doses de 0 t ha ${ }^{-1}$ e 60 t ha $^{-1}$ de composto orgânico, respectivamente.

PALAVRAS-CHAVE: Jatropha curcas L.; biocombustível; Glomus clarum; qualidade de mudas.

oleaginosas utilizadas no mercado de biocombustíveis (Arruda et al. 2004). O pinhão manso é de fácil cultivo e seu óleo tem variações pouco significativas de acidez, além de possuir maior estabilidade à oxidação do que culturas como a soja (Glycine max) e a palma (Elaeis guineensis), e baixa viscosidade, quando comparada à da mamona (Ricinus communis) (Achten et al. 2008).

1. Trabalho recebido em maio/2009 e aceito para publicação em set./2010 (n registro: PAT 6303/ DOI: 10.5216/pat.v40i3.6303).

2. Universidade Estadual do Mato Groso do Sul (UEMS), Unidade Universitária de Aquidauana, Departamento de Solos, Aquidauana, MS, Brasil.E-mails: schiavo@uems.br, cris_agro07@hotmail.com,jrrosset@hotmail.com, mateussecretti@hotmail.com, ricelly1@hotmail.com,nccappi@uems.br. 
A espécie se propaga por via seminal e vegetativa (Kochhar et al. 2008). Plantios desuniformes, com uso de sementes, e a baixa demanda de material vegetal (estacas) têm sido apontados como os principais fatores que limitam a expansão da cultura (Openshaw 2000). Assim, pesquisas com enfoque na melhoria da qualidade das plantas, principalmente na fase de produção de mudas, são imprescindíveis.

A obtenção de mudas de pinhão manso, com alta qualidade morfofisiológica, produzidas a baixo custo, é um dos fatores mais importantes para o sucesso do seu desenvolvimento no campo. Neste sentido, os fungos micorrízicos arbusculares (FMAs) podem ter efeito positivo, uma vez que apresentam a capacidade de aumentar a absorção de nutrientes, principalmente aqueles de baixa mobilidade no solo, como o P (Nogueira \& Cardoso 2000), e, ainda, conseguem reter, no micélio, elementos que se encontram em níveis tóxicos, como os encontrados em alguns fertilizantes orgânicos (Soares et al. 2007).

As áreas urbanas caracterizam-se pela alta produção de resíduos orgânicos, tanto de origem domiciliar quanto de áreas comerciais e industriais, como padarias, açougues e frigoríficos. A destinação final destes resíduos pode ser a incineração, a temperaturas acima de $900^{\circ} \mathrm{C}$, e o descarte, em aterros ou terrenos baldios. Estas práticas têm gerado crescente preocupação na população, devido ao risco ambiental que representam. Uma alternativa é a utilização agrícola destes resíduos, visando ao condicionamento físico e químico dos solos. Para tanto, é necessário o tratamento destes resíduos, a fim de garantir um produto estabilizado e de boa qualidade, que forneça nutrientes ao solo, de forma equilibrada.

O composto orgânico denominado Organosuper $^{\circledR}$ têm sido utilizado na agricultura, principalmente como fonte de nitrogênio $(\mathrm{N})$ e fósforo $(\mathrm{P})$. Ele é produzido utilizando-se excrementos sólidos e líquidos de origem animal, misturados a restos vegetais de composição variada. Posteriormente, o resíduo é inoculado com bactérias catalizadoras e passa por processo de compostagem, a fim de tornar o composto adequado ao uso agrícola.

Neste contexto, o presente trabalho teve como objetivo avaliar o efeito da adição de doses de composto orgânico (Organosuper ${ }^{\circledR}$ ) e da presença ou ausência do fungo micorrízico arbuscular Glomus clarum sobre o crescimento e absorção de fósforo $(\mathrm{P})$ e nitrogênio $(\mathrm{N})$, em mudas de pinhão manso (Jatropha curcas L.).

\section{MATERIAL E MÉTODOS}

O experimento foi desenvolvido em casa-devegetação, na Unidade Universitária de Aquidauana da Universidade Estadual do Mato Grosso do Sul (UEMS), localizada entre as coordenadas $20^{\circ} 27^{\prime} 20^{\prime \prime}$ de latitude S e 55040'17" de longitude W. Segundo Köppen, o clima da região é do tipo Aw, classificado como tropical-quente, subúmido, com precipitação pluviométrica anual variando de $1.200 \mathrm{~mm}$ a $1.400 \mathrm{~mm}$, com período chuvoso bem definido, de outubro a março, e período seco de abril a setembro. A temperatura média anual é de $24^{\circ} \mathrm{C}$, com máxima diária de $36^{\circ} \mathrm{C}$, durante a primavera, e mínimas de $12^{\circ} \mathrm{C}$, no inverno.

O substrato utilizado no experimento foi constituído por uma mistura 1/1 (v/v) de vermiculita, com o horizonte subsuperficial de um solo classificado como Argissolo Vermelho-Amarelo distroférrico (Embrapa 2006). O substrato foi duplamente esterilizado em autoclave, a $121^{\circ} \mathrm{C}$, por uma hora, e, após esterilização, foi colocado em vasos plásticos de $5 \mathrm{dm}^{3}$ de capacidade. Foram adicionadas doses do composto orgânico, considerando-se o volume de substrato ocupado em cada vaso, tendo como base o volume de solo de 1 hectare, com $20 \mathrm{~cm}$ de profundidade. Dessa maneira, as doses foram equivalentes a $0 \mathrm{t} \mathrm{ha}^{-1}, 15 \mathrm{t} \mathrm{ha}^{-1}, 30 \mathrm{t} \mathrm{ha}^{-1}, 60 \mathrm{t} \mathrm{ha}^{-1} \mathrm{e} 120 \mathrm{t} \mathrm{ha}^{-1}$. A análise química do composto orgânico, bem como do solo utilizado como substrato, encontram-se na Tabela 1.

O delineamento experimental utilizado foi o de blocos ao acaso, em esquema fatorial $5 \times 2$, sendo os fatores as doses de composto orgânico Organosu$\operatorname{per}^{\circledR}\left(0 \mathrm{tha}^{-1}, 15 \mathrm{tha}^{-1}, 30 \mathrm{tha}^{-1}, 60 \mathrm{t} \mathrm{ha}^{-1}\right.$ e $\left.120 \mathrm{tha}^{-1}\right)$ e o tratamento microbiológico com a presença de FMAs (inoculado com Glomus clarum) e ausência (sem inoculação com Glomus clarum), com quatro repetições.

As sementes de pinhão manso foram desinfectadas superficialmente, pela imersão em hipoclorito de sódio a $10 \%$, por três minutos. Posteriormente, foram lavadas com água esterilizada, para remoção do excesso de hipoclorito de sódio, colocadas em placa de Petri com algodão umedecido e levadas ao germinador, com temperatura de $25^{\circ} \mathrm{C}$ e umidade relativa controlada de $85 \%$, até a emissão das radículas. A semeadura nos vasos foi realizada colocando-se quatro sementes prégerminadas, efetudando-se, posteriormente, desbaste, permanecendo duas plantas por vaso. 
Tabela 1. Características químicas do composto orgânico $\left(\right.$ Organosuper $\left.^{\mathbb{R}}\right)$ e do solo utilizado no experimento.

\begin{tabular}{|c|c|c|}
\hline \multirow{2}{*}{ Características } & \multicolumn{2}{|c|}{ Material utilizado } \\
\hline & Organosuper $^{\circledR}$ & Solo \\
\hline $\mathrm{pH}^{1}$ & 7.60 & 5,60 \\
\hline $\operatorname{MO}\left(\mathrm{g} \mathrm{dm}^{-3}\right)^{2}$ & 495,60 & 13,80 \\
\hline $\mathrm{P}\left(\mathrm{mg} \mathrm{dm}^{-3}\right)^{3}$ & 16,60 & 10,00 \\
\hline $\mathrm{K}^{+}\left(\mathrm{mmol} \mathrm{dm}^{-3}\right)^{4}$ & 76,72 & 5,40 \\
\hline $\mathrm{Ca}^{+2}\left(\mathrm{mmol} \mathrm{dm}^{-3}\right)^{5}$ & 942,64 & 21,00 \\
\hline $\operatorname{Mg}^{+2}\left(\mathrm{mmol} \mathrm{dm}^{-3}\right)^{5}$ & 218,11 & 9,00 \\
\hline $\mathrm{Al}^{+3}\left(\mathrm{mmol}^{\mathrm{c}} \mathrm{dm}^{-3}\right)^{6}$ & - & 1,00 \\
\hline $\mathrm{H}+\mathrm{Al}\left(\mathrm{mmol} \mathrm{dm}^{-3}\right)^{7}$ & - & 33,00 \\
\hline $\mathrm{SB}\left(\mathrm{mmol} \mathrm{dm}^{-3}\right)$ & $1.237,47$ & 35,40 \\
\hline CTC $\left(\mathrm{mmol} \mathrm{dm}^{-3}\right)$ & - & 68,40 \\
\hline $\mathrm{V}(\%)$ & - & 52,00 \\
\hline$m(\%)$ & - & 2,80 \\
\hline $\mathrm{B}\left(\mathrm{mg} \mathrm{dm}^{-3}\right)^{8}$ & 220,00 & - \\
\hline $\mathrm{Cu}\left(\mathrm{mg} \mathrm{dm}^{-3}\right)^{9}$ & 100,00 & - \\
\hline $\mathrm{Fe}\left(\mathrm{mg} \mathrm{dm}^{-3}\right)^{9}$ & $29.800,00$ & - \\
\hline $\left.\operatorname{Mn}(\mathrm{mg} \mathrm{dm})^{-3}\right)^{9}$ & 530,00 & - \\
\hline $\mathrm{Zn}\left(\mathrm{mg} \mathrm{dm}^{-3}\right)^{9}$ & 40,00 & - \\
\hline
\end{tabular}

${ }^{1}$ Obtido em água (relação solo:solução 1:2,5); ${ }^{2}$ carbono orgânico total (oxidação pelo dicromato de potássio, em meio sulfúrico). $\mathrm{MO}=$ carbono orgânico $\mathrm{x}$ 1,724; ${ }^{3} \mathrm{P}$ disponível (extraído com solução de Mehlich-1 e determinado por colorimetria); ${ }^{4}$ extraídos por Mehlich-1 e determinados por espectrofotometria de chama; ${ }^{5}$ extraídos com KCL $1 \mathrm{~mol} \mathrm{~L}^{-1}$ e determinados por complexiometria; ${ }^{6}$ extraído com solução de KCL 1 mol L ${ }^{-1}$ e determinado por titulação; ${ }^{7}$ extraídos com solução de acetato de cálcio $\left(0,5 \mathrm{~mol} \mathrm{~L}^{-1}\right)$ e determinados por titulação; ${ }^{8}$ extraído com água quente e dosado com azometina-H; ${ }^{9}$ digestão nitro-perclórica e determinados por espectrofotometria de absorção atômica.

A inoculação com FMA foi realizada no momento da semeadura, tendo como inóculo $5 \mathrm{~mL}$ de uma mistura de solo, raízes colonizadas e esporos do FMA Glomus clarum. Para equilibrar a microbiota, entre os tratamentos inoculados e sem inoculação, foram adicionados aos últimos, depois da semeadura, $1 \mathrm{~mL}$ de filtrado de inóculo isento de propágulos de FMAs.

O crescimento das mudas de pinhão manso foi avaliado com medições periódicas de altura de mudas e diâmetro à altura do colo $(30,60,90$ e 120 dias após a semeadura - DAS). Ao término do experimento (120 DAS), as mudas de cada tratamento foram coletadas e o sistema radicular separado da parte aérea, para determinação da colonização micorrízica. Após lavagem, subamostras de $2 \mathrm{~cm}$ de comprimento de raízes foram coletadas e conservadas em etanol, a 50\%, para posterior determinação da colonização, pelo método da interseção em placa de Petri reticulada (Giovannetti \& Mosse 1980), após a coloração das raízes com azul de metila (Koske \& Gemma 1989). Para determinação da matéria seca total da planta e dos teores de $\mathrm{N}$ e $\mathrm{P}$, a parte aérea e o sistema radicular foram levados para estufa, $\mathrm{a} 75^{\circ} \mathrm{C}$, por 48 horas. A seguir, o material foi pesado, para determinação da matéria seca, moído em moinho tipo Willey, passado em peneira de 20 mesh e armazenado em frascos hermeticamente fechados. Após o material ser submetido à digestão sulfúrica (para determinação de $\mathrm{N}$ ) e nítricoperclórica para $\mathrm{P}, \mathrm{o} \mathrm{N}$ foi determinado pelo método de Nessler (Jackson 1965) e o $\mathrm{P}$ foi determinado por colorimetria, pelo método do molibdato (Malavolta et al. 1997).

A partir da matéria seca total, de acordo com Plenchette et al. (1983), determinou-se a dependência micorrízica (DM) e eficiência micorrízica (EM), de acordo com o seguinte cálculo: $\mathrm{DM}=$ (matéria seca de mudas micorrizadas) - (matéria seca de mudas não micorrizadas)/ matéria seca de mudas micorrizadas $\mathrm{x}$ 100; e EM = (matéria seca de mudas micorrizadas) (matéria seca de mudas não micorrizadas)/ matéria seca de mudas não micorrizadas x 100.

Os dados obtidos foram submetidos a análise de variância e as médias dos tratamentos de inoculação por Glomus clarum e as doses de composto orgânico a comparação de médias, pelo teste de Tukey, a $5 \%$, e análise de regressão, respectivamente.

\section{RESULTADOS E DISCUSSÃO}

As raízes das plantas não inoculadas com o FMA Glomus clarum apresentaram-se isentas de colonização (Tabela 2). A colonização radicular das mudas de pinhão manso que receberam inóculo do endófito Glomus clarum foi crescente, em função das doses de composto orgânico aplicadas, com valores de $62,5 \%$ a $90 \%$, para as doses de $0 \mathrm{t} \mathrm{ha}^{-1}$ e $120 \mathrm{tha}^{-1}$, respectivamente (Tabela 2). Carvalho (2008), em diferentes áreas de plantio com pinhão manso, no Estado de Minas Gerais, verificou elevada taxa de colonização, variando de 75,7\% a 97,4\%, mesmo em condições de elevada fertilidade do solo, sugerindo alta dependência micorrízica desta espécie. Os valores encontrados nas maiores doses de composto orgânico enquadram-se nesta variação.

Cabe ressaltar que a percentagem de colonização nem sempre é uma característica segura para se definir o efeito que o endófito causa no crescimento de sua planta hospedeira. Em algumas plantas, taxas tão baixas, como $5 \%$, já foram suficientes para um bom desenvolvimento (Karanika et al. 2008). Por outro lado, alguns fungos têm a habilidade de se associar às raízes indiscriminadamente, produzindo efeitos que variam de neutros a negativos para as plantas, sendo, por isto, denominados oportunistas ou comensalistas (Larsen et al. 2009). Destaca-se 
Tabela 2. Colonização micorrízica, altura e diâmetro à altura do colo de mudas de pinhão manso, inoculadas com fungo micorrízico arbuscular Glomus clarum, sob doses de composto orgânico.

\begin{tabular}{|c|c|c|c|c|c|c|c|}
\hline \multirow{2}{*}{ Inoculação ${ }^{1}$} & \multicolumn{5}{|c|}{ Doses de composto orgânico - t ha $^{-1}\left(\right.$ Organosuper $\left.^{\circledR}\right)$} & \multirow{2}{*}{$\begin{array}{c}\text { Regressão } \\
\mathrm{R}^{2}\end{array}$} & \multirow{2}{*}{ Equação } \\
\hline & 0 & 15 & 30 & 60 & 120 & & \\
\hline \multicolumn{8}{|c|}{ Colonização micorrízica (\%) } \\
\hline $\mathrm{GC}$ & 62,5 & 67,5 & 75 & 77,5 & 90,0 & - & - \\
\hline $\mathrm{C}$ & 0 & 0 & 0 & 0 & 0 & - & - \\
\hline \multicolumn{8}{|c|}{ Altura das plantas, 30 dias após semeadura $(\mathrm{cm})$} \\
\hline GC & $14,55 \mathrm{a}$ & $15,05 \mathrm{a}$ & $16,30 \mathrm{a}$ & $14,25 \mathrm{a}$ & $15,25 \mathrm{a}$ & - & - \\
\hline $\mathrm{C}$ & $16,10 \mathrm{a}$ & $15,90 \mathrm{a}$ & $15,52 \mathrm{a}$ & $12,95 \mathrm{a}$ & $15,50 \mathrm{a}$ & - & - \\
\hline \multicolumn{8}{|c|}{ Altura das plantas, 60 dias após semeadura $(\mathrm{cm})$} \\
\hline $\mathrm{GC}$ & $15,13 \mathrm{a}$ & $15,62 \mathrm{a}$ & $17,00 \mathrm{a}$ & $15,13 \mathrm{a}$ & $16,50 \mathrm{a}$ & - & - \\
\hline $\mathrm{C}$ & $16,50 \mathrm{a}$ & $17,75 \mathrm{a}$ & $16,50 \mathrm{a}$ & $15,13 \mathrm{a}$ & $17,12 \mathrm{a}$ & - & - \\
\hline \multicolumn{8}{|c|}{ Altura das plantas, 90 dias após semeadura $(\mathrm{cm})$} \\
\hline GC & $15,75 \mathrm{a}$ & $16,00 \mathrm{a}$ & $17,87 \mathrm{a}$ & $15,87 \mathrm{a}$ & $18,87 \mathrm{a}$ & - & - \\
\hline $\mathrm{C}$ & $17,12 \mathrm{a}$ & $19,37 \mathrm{a}$ & $17,63 \mathrm{a}$ & $17,50 \mathrm{a}$ & $18,62 \mathrm{a}$ & - & - \\
\hline \multicolumn{8}{|c|}{ Altura das plantas, 120 dias após semeadura (cm) } \\
\hline $\mathrm{GC}$ & $16,25 \mathrm{a}$ & $16,62 \mathrm{~b}$ & $18,25 \mathrm{a}$ & $16,25 \mathrm{a}$ & $18,50 \mathrm{a}$ & - & - \\
\hline $\mathrm{C}$ & $17,37 \mathrm{a}$ & $20,00 \mathrm{a}$ & $17,75 \mathrm{a}$ & $17,75 \mathrm{a}$ & $19,25 \mathrm{a}$ & - & - \\
\hline \multicolumn{8}{|c|}{ Diâmetro à altura do colo das plantas, 30 dias após semeadura (mm) } \\
\hline $\mathrm{GC}$ & $7,65 \mathrm{a}$ & $6,72 \mathrm{a}$ & $6,75 \mathrm{a}$ & $7,81 \mathrm{a}$ & $8,02 \mathrm{a}$ & - & - \\
\hline $\mathrm{C}$ & $7,83 \mathrm{a}$ & $7,56 \mathrm{a}$ & $7,85 \mathrm{a}$ & $6,68 \mathrm{a}$ & $8,43 \mathrm{a}$ & - & - \\
\hline \multicolumn{8}{|c|}{ Diâmetro à altura do colo das plantas, 60 dias após semeadura (mm) } \\
\hline $\mathrm{GC}$ & $11,75 \mathrm{a}$ & $11,50 \mathrm{a}$ & $13,63 \mathrm{a}$ & $13,75 \mathrm{a}$ & $18,00 \mathrm{a}$ & $\mathrm{L}, 0,94^{*}$ & $\hat{\mathrm{y}}=11,33+0,05312 * x$ \\
\hline $\mathrm{C}$ & $10,50 \mathrm{a}$ & $12,25 \mathrm{a}$ & $11,25 \mathrm{~b}$ & $13,00 \mathrm{a}$ & $13,50 \mathrm{~b}$ & - & - \\
\hline \multicolumn{8}{|c|}{ Diâmetro à altura do colo das plantas, 90 dias após semeadura (mm) } \\
\hline $\mathrm{GC}$ & $13,25 \mathrm{a}$ & $12,50 \mathrm{~b}$ & $14,75 \mathrm{a}$ & $16,25 \mathrm{a}$ & $19,75 \mathrm{a}$ & $\mathrm{L}, 0,95^{*}$ & $\hat{\mathrm{y}}=12,64+0,05916 * \mathrm{x}$ \\
\hline $\mathrm{C}$ & $13,50 \mathrm{a}$ & $15,50 \mathrm{a}$ & $15,75 \mathrm{a}$ & $17,25 \mathrm{a}$ & $20,00 \mathrm{a}$ & $\mathrm{L}, 0,96^{*}$ & $\hat{\mathrm{y}}=14,15+0,05000 * x$ \\
\hline \multicolumn{8}{|c|}{ Diâmetro à altura do colo das plantas, 120 dias após semeadura (mm) } \\
\hline GC & $14,00 \mathrm{a}$ & $14,75 \mathrm{~b}$ & $17,25 \mathrm{a}$ & $18,75 \mathrm{a}$ & $20,75 \mathrm{a}$ & $\mathrm{L}, 0,92 *$ & $\hat{\mathrm{y}}=14,57+0,05625 * \mathrm{x}$ \\
\hline $\mathrm{C}$ & $15,63 \mathrm{a}$ & $17,50 \mathrm{a}$ & $17,25 \mathrm{a}$ & $19,50 \mathrm{a}$ & $21,25 \mathrm{a}$ & $\mathrm{L}, 0,93^{*}$ & $\hat{\mathrm{y}}=16,23+0,04437 * \mathrm{x}$ \\
\hline
\end{tabular}

que, nem sempre, o estabelecimento da associação simbiótica garante eficiência micorrízica elevada. Muitas vezes, as espécies de FMAs com mecanismos evoluídos em infectividade não se apresentam especializadas em fornecer benefício para a planta, ou seja, são ineficientes nas trocas, não favorecendo o crescimento da planta hospedeira (Sena et al. 2004). Dentre vários fatores, a colonização radicular pode ser afetada pelo $\mathrm{pH}$ e pela concentração de nutriente do solo (Koide \& Li 1990). De maneira geral, a acidez e a baixa disponibilidade de nutrientes favorecem a colonização radicular, indicando que o fungo, ao mesmo tempo em que beneficia o crescimento das plantas, aumenta a atividade metabólica nas raízes. Entretanto, em situações de alta disponibilidade de nutrientes, especialmente de $\mathrm{P}$, as plantas tendem a diminuir a colonização (Covacevich et al. 2006).

$\mathrm{O}$ crescimento de pinhão manso avaliado pela altura das mudas não apresentou ajuste de regressão, em função das doses de composto orgânico aplicado, sendo, também, pouco expressivo seu crescimento, em relação à época de avaliação (Tabela 2). Ainda, não observou-se efeito da inoculação com Glomus clarum, exceto na dose de $15 \mathrm{t} \mathrm{ha}^{-1}$, aos 120 DAS. Por outro lado, em todas as épocas de avaliação, houve crescimento linear do diâmetro à altura do colo das mudas inoculadas com Glomus clarum, em função das doses de composto orgânico, exceto aos 30 DAS. No tratamento sem inoculação, houve crescimento linear para diâmetro à altura do colo apenas nas avaliações realizadas aos 90 DAS e 120 DAS. Verificou-se, também, que o diâmetro à altura do colo, aos 60 DAS, nas doses de $30 \mathrm{tha}^{-1}$ e $120 \mathrm{t} \mathrm{ha}^{-1}$, foi maior nas mudas inoculadas com Glomus clarum. No entanto, aos 90 DAS e 120 DAS, na dose de $15 \mathrm{tha}^{-1}$, tal comportamento inverteu-se, ou seja, mudas do tratamento controle tiveram maior diâmetro à altura do colo.

Houve acúmulo crescente de matéria seca da parte aérea (MSA), raízes (MSR) e planta inteira (MST) apenas com inoculação de Glomus clarum (Tabela 3). Especificamente na dose de $15 \mathrm{t} \mathrm{ha}^{-1}$ 
Tabela 3. Matéria seca de pinhão manso acumulada nas folhas (MSF), caules (MSC), parte aérea (MSA), raízes (MSR) e total (MST), com inoculação do fungo micorrízico arbuscular Glomus clarum, sob doses de composto orgânico.

\begin{tabular}{|c|c|c|c|c|c|c|c|}
\hline \multirow{2}{*}{ Inoculação $^{1}$} & \multicolumn{5}{|c|}{ Doses de composto orgânico - t ha $^{-1}\left(\right.$ Organosuper $\left.^{\mathbb{B}}\right)$} & \multirow{2}{*}{$\begin{array}{c}\text { Regressão } \\
\mathrm{R}^{2} \\
\end{array}$} & \multirow{2}{*}{ Equação } \\
\hline & 0 & 15 & 30 & 60 & 120 & & \\
\hline & & & & $M S F(g)$ & & & \\
\hline GC & $0,81 \mathrm{a}$ & $1,44 \mathrm{~b}$ & $1,65 \mathrm{a}$ & $1,43 \mathrm{a}$ & $2,11 \mathrm{a}$ & - & - \\
\hline $\mathrm{C}$ & $0,98 \mathrm{a}$ & $2,76 \mathrm{a}$ & $1,95 \mathrm{a}$ & $\begin{array}{l}1,98 \mathrm{a} \\
M S C(g)\end{array}$ & $2,73 \mathrm{a}$ & - & - \\
\hline$\underset{\mathrm{C}}{\mathrm{GC}}$ & $\begin{array}{l}4,18 \mathrm{a} \\
4,76 \mathrm{a}\end{array}$ & $\begin{array}{l}4,60 \mathrm{~b} \\
8,09 \mathrm{a}\end{array}$ & $\begin{array}{l}6,10 \mathrm{a} \\
6,71 \mathrm{a}\end{array}$ & $\begin{array}{l}7,06 \mathrm{a} \\
7,82 \mathrm{a} \\
M S A(g)\end{array}$ & $\begin{array}{l}10,52 \mathrm{a} \\
10,51 \mathrm{a}\end{array}$ & $\begin{array}{c}\mathrm{L}, 0,99^{*} \\
-\end{array}$ & $\hat{\mathrm{y}}=4,11+0,0530167^{*} \mathrm{x}$ \\
\hline$\underset{\mathrm{C}}{\mathrm{GC}}$ & $\begin{array}{l}5,00 \mathrm{a} \\
5,74 \mathrm{a}\end{array}$ & $\begin{array}{c}6,04 \mathrm{~b} \\
10,84 \mathrm{a}\end{array}$ & $\begin{array}{l}7,75 \mathrm{a} \\
8,66 \mathrm{a}\end{array}$ & $\begin{array}{l}8,50 \mathrm{a} \\
9,81 \mathrm{a} \\
M S R(g)\end{array}$ & $\begin{array}{l}12,63 \mathrm{a} \\
13,23 \mathrm{a}\end{array}$ & $\begin{array}{c}\mathrm{L}, 0,98^{*} \\
-\end{array}$ & $\hat{y}=5,22+0,0613917 * x$ \\
\hline $\mathrm{GC}$ & $1,73 \mathrm{a}$ & $1,78 \mathrm{~b}$ & $2,50 \mathrm{a}$ & $2,58 \mathrm{a}$ & $3,76 \mathrm{a}$ & $\mathrm{L}, 0,95^{*}$ & $\hat{y}=1,71+0,0169125^{*} x$ \\
\hline $\mathrm{C}$ & $1,68 \mathrm{a}$ & $3,48 \mathrm{a}$ & $2,78 \mathrm{a}$ & $\begin{array}{l}3,80 \mathrm{a} \\
\quad M S T \text { (g) }\end{array}$ & $4,75 \mathrm{a}$ & & \\
\hline $\mathrm{GC}$ & $6,72 \mathrm{a}$ & $7,83 \mathrm{~b}$ & $10,26 \mathrm{a}$ & $11,07 \mathrm{a}$ & 16,39 a & $\mathrm{L}, 0,97^{*}$ & $\hat{y}=6,93+0,0783042 * x$ \\
\hline $\mathrm{C}$ & $7,42 \mathrm{a}$ & $14,33 \mathrm{a}$ & $11,44 \mathrm{a}$ & $13,61 \mathrm{a}$ & $17,98 \mathrm{a}$ & - & - \\
\hline
\end{tabular}

Médias seguidas da mesma letra não diferem entre si, em tratamentos de inoculação, pelo teste Tukey, a 5\% de probabilidade.

${ }^{1} \mathrm{GC}$ : Mudas inoculadas com a espécie de fungo micorrízico Glomus clarum; C: mudas sem nenhuma inoculação.

${ }^{2} \mathrm{~L}$ : regressão linear.

* Significativo a $1 \%$ de probabilidade.

de composto orgânico, as mudas inoculadas com Glomus clarum tiveram os menores acúmulos de matéria seca.

A baixa resposta, ou até mesmo o crescimento inferior de mudas de pinhão manso inoculadas com Glomus clarum, verificado no presente trabalho, possivelmente, podem ser atribuídos aos elevados teores de nutrientes, especificamente de P, presentes no composto orgânico (Tabela 1). De maneira geral, plantas micorrizadas alcançam seu desenvolvimento máximo em pequenas doses de $\mathrm{P}$, sendo tal resposta característica intrínseca, de herança genética, relacionada às características morfológicas e fisiológicas do hospedeiro, que controlam a demanda e suprimento de P, e, assim, o grau de dependência da planta (Koide 1991). Com base nisto, o teor de P no solo mais apropriado para resposta à micorriza é altamente variável, entre as espécies e genótipos, havendo diferenças, mesmo em plantas muito próximas geneticamente (Clement \& Habte 1995). O comportamento decrescente no acúmulo da matéria seca, com aumento das doses de $\mathrm{P}$, foi, repetidamente, descrito na literatura, para as mais variadas plantas (Melloni et al. 2000, Nogueira \& Cardoso 2000, Moreira-Souza \& Cardoso 2002).

Para o tratamento controle, os teores de $\mathrm{P}$, na parte aérea de mudas de pinhão manso, apresentaram ajuste quadrático, em resposta à adição de doses de composto orgânico (Tabela 4). A inoculação com Glomus clarum proporcionou, entre as doses de $15 \mathrm{t} \mathrm{ha}^{-1}$ a $120 \mathrm{t} \mathrm{ha}^{-1}$, aumentos nos teores de $\mathrm{P}$, na parte aérea. Quanto ao conteúdo de P na parte aérea, para ambos os tratamentos, verificou-se ajuste linear, em função das doses de composto orgânico aplicado, porém, apenas na maior dose de composto orgânico $\left(120 \mathrm{t} \mathrm{ha}^{-1}\right)$ foram observados incrementos, devido à inoculação com Glomus clarum.

Os teores de N, na parte aérea de mudas de pinhão manso, não apresentaram ajuste de regressão, para as doses de composto orgânico aplicado, sendo observado efeito positivo da inoculação com Glomus clarum apenas nas doses de $0 \mathrm{t} \mathrm{ha}^{-1}$ e $60 \mathrm{t} \mathrm{ha}^{-1}$ (Tabela 4). Com relação ao conteúdo de $\mathrm{N}$ na parte aérea, apenas no tratamento inoculado com Glomus clarum verificou-se aumento linear, em função das doses de composto orgânico aplicado.

Efeitos positivos da inoculação com FMA sobre o crescimento e utilização de P são comuns na literatura, resultantes da ação do micélio externo às raízes, que aumenta a superfície de absorção de água e de nutrientes, principalmente o P (Moreira \& Siqueira 2002). Portanto, para o pinhão manso, poucas informações têm sido apresentadas sobre os efeitos dos FMAs sobre o crescimento e nutrição. Carvalho (2008), em levantamento de FMAs em diferentes áreas com cultivo de pinhão manso, no Estado de Minas Gerais, observou elevada colonização micorrízica $(75,7 \%$ a $97,4 \%)$ e densidade de esporos. O mesmo autor verificou que a elevada fertilidade natural do solo de algumas áreas estudadas não inibiu a colonização micorrízica das plantas, sugerindo alta dependência micorrízica da espécie. 
Tabela 4. Teores e conteúdos de fósforo $(\mathrm{P})$ e nitrogênio $(\mathrm{N})$ da parte aérea de pinhão manso, inoculada com o fungo micorrízico arbuscular Glomus clarum, sob doses de composto orgânico.

\begin{tabular}{|c|c|c|c|c|c|c|c|}
\hline \multirow{2}{*}{ Inoculação $^{1}$} & \multicolumn{5}{|c|}{ Doses de composto orgânico - t ha $^{-1}\left(\right.$ Organosuper $\left.^{\circledR}\right)$} & \multirow{2}{*}{$\begin{array}{c}\text { Regressão } 0^{2} \\
\mathrm{R}^{2}\end{array}$} & \multirow{2}{*}{ Equação } \\
\hline & 0 & 15 & 30 & 60 & 120 & & \\
\hline \multicolumn{8}{|c|}{ Teor de $P\left(g \mathrm{~kg}^{-1}\right)$} \\
\hline GC & $1,38 \mathrm{a}$ & $2,35 \mathrm{a}$ & $1,82 \mathrm{a}$ & $2,37 \mathrm{a}$ & $2,59 \mathrm{a}$ & - & - \\
\hline $\mathrm{C}$ & $1,35 \mathrm{a}$ & $1,22 \mathrm{~b}$ & $1,33 \mathrm{~b}$ & $1,51 \mathrm{~b}$ & $1,72 \mathrm{~b}$ & $\mathrm{Q}, 0,93^{*}$ & $\hat{y}=1,34+0,040979 \sqrt{x}+0,0071893 * x$ \\
\hline \multicolumn{8}{|c|}{ Conteúdo de P (mg planta ${ }^{-1}$ parte aérea $)$} \\
\hline GC & $6,90 \mathrm{a}$ & $13,63 \mathrm{a}$ & $14,24 \mathrm{a}$ & $19,99 \mathrm{a}$ & $32,85 \mathrm{a}$ & $\mathrm{L}, 0,98^{*}$ & $\hat{y}=8,37+0,203396 * x$ \\
\hline \multicolumn{8}{|c|}{ Teor de $N\left(\mathrm{~g} \mathrm{~kg}^{-1}\right)$} \\
\hline$\underset{\mathrm{C}}{\mathrm{GC}}$ & $\begin{array}{l}36,33 \mathrm{a} \\
24,22 \mathrm{~b}\end{array}$ & $\begin{array}{l}36,95 \mathrm{a} \\
44,73 \mathrm{a}\end{array}$ & $\begin{array}{l}33,54 \mathrm{a} \\
28,95 \mathrm{a}\end{array}$ & $\begin{array}{l}40,82 \mathrm{a} \\
28,16 \mathrm{~b}\end{array}$ & $\begin{array}{l}37,07 \mathrm{a} \\
33,51 \mathrm{a}\end{array}$ & - & - \\
\hline \multicolumn{8}{|c|}{ Conteúdo de $N$ (mg planta-1 parte aérea) } \\
\hline $\mathrm{GC}$ & $183,12 \mathrm{a}$ & $227,01 \mathrm{~b}$ & $260,62 \mathrm{a}$ & 343,26 a & $466,95 \mathrm{a}$ & $\mathrm{L}, 0,99^{*}$ & $\hat{y}=190,15+2,35665 * x$ \\
\hline $\mathrm{C}$ & 136,55 a & 498,69 a & 255,78 a & 276,39 a & 437,99 a & & \\
\hline
\end{tabular}

Médias seguidas da mesma letra não diferem entre si, em tratamentos de inoculação, pelo teste Tukey, a $5 \%$ de probabilidade.

${ }^{1} \mathrm{GC}$ : Mudas inoculadas com a espécie de fungo micorrízico Glomus clarum; C: mudas sem nenhuma inoculação.

${ }^{2} \mathrm{~L}$ : regressão linear; Q: quadrática.

* Significativo a $1 \%$ de probabilidade.

No presente trabalho, foi verificada baixa, ou até mesmo negativa, dependência micorrízica (DM) e eficiência micorrízica (EM) (Figuras 1a e 1b), ou seja, as plantas inoculadas com Glomus clarum apresentaram menor acúmulo de matéria seca, em relação ao tratamento controle, podendo caracterizar a interação FMA x hospedeiro como parasítica. Tal fato pode ser atribuído aos elevados teores de $\mathrm{P}$ presentes no composto orgânico (Tabela 1). Este comportamento confirma a maior micorrização, em condições de dose de $\mathrm{P}$ abaixo da ótima para o crescimento vegetal (Melloni et al. 2000, Moreira \& Siqueira 2002), ou seja, a dependência micorrízica é inversamente proporcional à disponibilidade de $\mathrm{P}$.

A baixa resposta do crescimento das mudas de pinhão manso à adubação orgânica e à inoculação com FMA, observada no presente trabalho, não constitui fator negativo. A associação micorrízica não substitui a adubação, mas aumenta a eficiência de utilização pelas plantas, principalmente para nutrientes pouco móveis, como o P (Miranda \& Miranda 1997).

Normalmente, a eficiência micorrízica está relacionada com a quantidade de micélio externo formado no solo. Certos fungos podem possuir grande capacidade de colonizar o hospedeiro, mas a produção de hifas externas (estrutura que permite a maior absorção de nutrientes) varia muito entre as espécies de FMAs (Majdi et al. 2008). A eficiência micorrízica também depende de outros fatores. Algumas associações fungo-hospedeiro, dependendo do genótipo da planta, da espécie e ecótipo do fungo e da disponibilidade de $\mathrm{P}$ no solo, podem proporcionar apenas um baixo crescimento vegetal, onde o balanço energético se torna desfavorável à planta, e o que era para se tornar simbiose se torna parasitismo (Smith \& Read 2008).

(a)

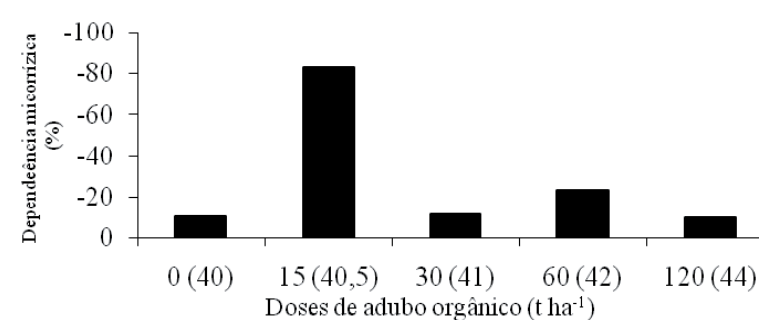

(b)

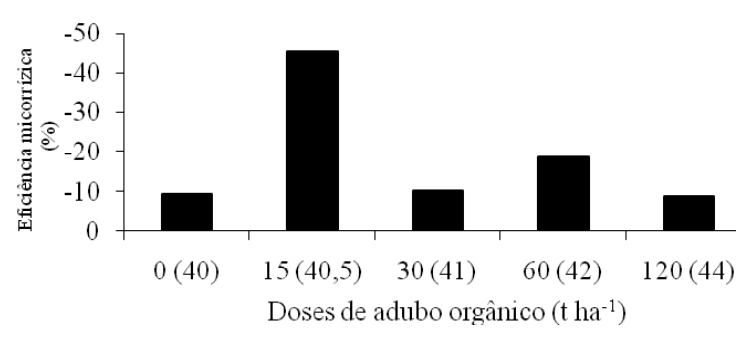

Figura 1. Dependência micorrízica (DM) (a) e eficiência micorrízica (EM) (b), em mudas de pinhão manso inoculadas com o fungo micorrízico arbuscular Glomus clarum, sob doses de composto orgânico. Valores entre parênteses, ao lado das doses de composto orgânico, referem-se aos teores aproximados de $\mathrm{P}$, em $\mathrm{g} \mathrm{kg}^{-1}$. 


\section{CONCLUSÕES}

1. De maneira geral, a aplicação de doses de composto orgânico proporcionou maior crescimento das mudas de pinhão manso, independentemente da inoculação com Glomus clarum.

2. Tanto a inoculação com Glomus clarum quanto a aplicação de composto orgânico proporcionaram às mudas de pinhão manso maior acúmulo de $\mathrm{P} \mathrm{e}$ $\mathrm{N}$, na parte aérea.

\section{REFERÊNCIAS}

ACHTEN, W. M. J. et al. Jatropha bio-diesel production and use. Biomass and Bioenergy, Oxford, v. 32, n. 12, p. 1063-1084, 2008.

ARRUDA, F. P. et al. Cultivo de pinhão-manso (Jatropha curcas L.) como alternativa para o semi-árido nordestino. Revista Brasileira de Oleagionosas e Fibrosas, Campina Grande, v. 8, n. 1, p. 789-799, 2004.

CARVALHO, A. M. X. Fungos micorrízicos arbusculares no crescimento de mudas de pinhão manso (Jatropha curcas L.). 2008. 76 f. Dissertação (Mestrado em Microbiologia Agrícola)-Universidade Federal de Viçosa, Viçosa, 2008.

CLEMENT, C. R.; HABTE, M. Genotypic variation in vesicular-arbuscular mycorrhizal dependence of the pejibaye palm. Journal of Plant Nutrition, New York, v. 18, n. 9, p. 1907-1916, 1995.

COVACEVICH, F.; MARINO, M. A.; ECHEVERRÍA, E. The phosphorus source determines the arbuscular mycorrhizal potential and the native mycorrhizal colonization of tall fescue and wheatgrass. European Journal of Soil Biology, Oxford, v. 42, n. 3, p. 127-138, 2006.

EMPRESA BRASILEIRA DE PESQUISA AGROPECUÁRIA (Embrapa). Centro Nacional de Pesquisa de Solos. Sistema Brasileiro de Classificação de Solos. Brasília, DF: Embrapa, 2006.

GIOVANNETTI, M.; MOSSE, B. An evaluation of techniques for measuring vesicular-arbuscular mycorrhizal infection in roots. New Phytologist, London, v. 84, n. 3 , p. 489-500, 1980.

JACKSON, M. L. Soil chemical analysis. New Jersey: Prentice Hall, 1965.

KARANIKA, E. D. et al. Arbuscular mycorrhizal fungi in Northern Greece and influence of soil resources on their colonization. Pedobiologia, Jena, v. 51, n. 6, p. 409-418, 2008.
KOCHHAR, S.; SINGH, S. P.; KOCHHAR, V. K. Effect of auxins and associated biochemical changes during clonal propagation of the biofuel plant Jatropha curcas. Biomass and Bioenergy, Oxford, v. 32, n. 12, p. 1136-1143, 2008.

KOIDE, R. T. Nutrient supply, nutrient demand and plant response to mycorrhizal infection. New Phytologist, London, v. 117, n. 3, p. 365-386, 1991.

KOIDE, R. T.; LI, M. On host regulation of the vesiculararbuscular mycorrhizal symbiosis. New Phytologist, London, v. 114, n. 1, p. 59-74, 1990.

KOSKE, R. E.; GEMMA, J. N. A modified procedure for staining roots to detect VA mycorrhizas. Mycology Research, Cambridge, v. 92, n. 4, p. 488-505, 1989.

LARSEN, J.; CORNEJO, P.; BAREA, J. M. Interactions between the arbuscular mycorrhizal fungus Glomus intraradices and the plant growth promoting rhizobacteria Paenibacillus polymyxa and P. macerans in the mycorrhizosphere of Cucumis sativus. Soil Biology and Biochemistry, Oxford, v. 41, n. 2, p. 286-292, 2009.

MAJDI, H. et al. Effects of slash retention and wood ash addition on fine root biomass and production and fungal mycelium in a Norway spruce stand in SW Sweden. Forest Ecology and Management, Dorchester, v. 255, n. 7, p. 2109-2117, 2008.

MALAVOLTA, E.; VITTI, G. C.; OLIVEIRA, S. A. Avaliação do estado nutricional das plantas: princípios e aplicações. 2. ed. Piracicaba: Potafos, 1997.

MELLONI, R. et al. Fósforo adicionado e fungos micorrízicos arbusculares no crescimento e nutrição mineral de limoeiro-cravo (Citrus limonia (L.) Osbeck). Revista Brasileira de Ciência do Solo, Viçosa, v. 24, n. 4, p. 767-775, 2000.

MOREIRA, F. M. S.; SIQUEIRA, J. O. Microbiologia e bioquímica do solo. Lavras: UFLa, 2002.

MOREIRA-SOUZA, M.; CARDOSO, E. J. B. N. Dependência micorrízica de Araucaria angustifolia (BERT.) O. KTZE. sob doses de fósforo. Revista Brasileira de Ciência do Solo, Viçosa, v. 26, n. 4, p. 905-912, 2002.

MIRANDA, J. C. C.; MIRANDA, L. N. Micorrizas arbusculares. In: VARGAS, M. A. T.; HUNGRIA, M. (Eds.). Biologia dos solos do Cerrado. Planaltina: Embrapa-CPAC, 1997. p. 67-123.

NOGUEIRA, M. A.; CARDOSO, E. J. B. N. Produção de micélio externo por fungos micorrízicos arbusculares e crescimento da soja em função de doses de fósforo. Revista Brasileira de Ciência do Solo, Viçosa, v. 24, n. 2, p. 329-338, 2000. 
OPENSHAW, K. A review of Jatropha curcas: an oil plant of unfulfilled promise. Biomass and Bioenergy, Oxford, v. 19, n. 1, p. 1-15, 2000.

PLENCHETTE, C.; FORTIN, J. A.; FURLAN, V. Growth responses of several plant species to mycorrhizae in a soil of moderate P-fertility. Plant and Soil, London, v. 70, n. 2, p. 199-209, 1983.

SENA, J. O. A.; LABATE, C. A.; CARDOSO, E. J. B. N. Caracterização fisiológica da redução de crescimento de mudas de citrus micorrizadas em altas doses de fósforo. Revista Brasileira de Ciência do Solo, Viçosa, v. 28, n. 5, p. 827-832, 2004.
SMITH, S. E.; READ, D. J. Mycorrhizal symbiosis. 3. ed. New York: Academic Press, 2008.

SOARES, C. R. F. S. et al. Nutrição fosfática e micorriza arbuscular na redução da toxicidade de cádmio em trema [Trema micrantha (L.) Blum.]. Revista Árvore, Viçosa, v. 31, n. 5, p. 783-792, 2007. 